\title{
A System Solution to Concurrent Process Planning and Workshop Control
}

\author{
B. C. Schmidt \\ IPH-Institut für Integrierte Produktion Hannover gem. GmbH \\ Hollerithallee 6, D-30419 Hannover, Germany \\ T.: +4951127976-42; F.: +4951127976-88; \\ emailschmidt@iipr00.ifw.uni-hannover.de
}

\begin{abstract}
:
This paper discusses current weak points in manufacturing planning and the need for more collaboration between process planning and scheduling. It then describes the approach and prototype developments of a new software system (COMPLAN) in which process planning and workshop scheduling are supported as collaborative tasks that operate on the same data base. The system uses net-shaped process plans with manufacturing alternatives to increase planning flexibility in the workshop. The system provides equally manual and automatic planning functions for process planning and scheduling and also provides an interactive interface to CAD.
\end{abstract}

Keywords

production management, concurrent engineering, process planning, workshop scheduling.

\section{INTRODUCTION}

„How to improve performance in industry“ is the key question for which manufacturing engineers have to continuously find new and innovative answers. In recent years the efforts focused on two improvement areas.

Business Improvement, which is mainly achieved through organisational changes. These changes are inspired by new concepts or visions such as Lean Production, Business Process Re-engineering, Fractal Factory, Concurrent Engineering or Segmentation and Decentralisation.

Production Improvement, which is mainly achieved through technical and organisational changes that are driven by technology. In this area the efforts aim at continuously improving the processing of technical information (e.g. CAD/CAM, FEM) and logistical information (ERP/MRP), improving the employed manufacturing technology (High-Speed Machining, FMS). The advances are mostly characterised by making effective use of newly available 
technology such as lasers or supercomputers or by making use of newly developed methods such as fuzzy logic or genetic algorithms.

New concepts and visions for business improvement have received great attention in the last four years. The existing enormous potential for improvement by organisational changes in corporations had been neglected. Great successes were achieved and are still achieved today by Lean Production, Business Re-engineering and Decentralisation. But industrial competitiveness will always also be determined by the performance of manufacturing.

Especially, the successful implementation of concurrent engineering concepts will require the development of new underpinning technology and enabling information systems. The idea of concurrent engineering must not only be applied to product development but to the entire chain from development to regular production. It must not only aim at performing tasks in parallel and thus shortening the time-to-market, but it must focus on making the individual tasks in manufacturing also collaborative to thus achieve a better overall performance.

\subsection{Weak points in manufacturing planning}

Several weak points can be identified in the current state-of-the-art in manufacturing planning in industry. An efficient interface between CAD data and computer-aided process planning functions is missing. Also it is required to introduce generative process planning systems to industrial applications. Currently, automated process planning is only possible for a limited spectrum of workpieces. An introduction of automated generative planning modules with limited capabilities in industry requires that they are integrated with existing commercial CAPP systems. Process planning also has to provide more routing flexibility to workshop control. That requires on the other hand that information on logistical constraints is provided to process planning. The increased cost pressure no longer allows it to leave process plans unchanged for a long period of time. Revision and improvement of process plans will become a continuous task. Consequently, the functionality of existing process planning systems also needs to be extended to make CAPP systems a powerful tool for cost management and for the evaluation of make-or-buy decisions. Scheduling systems will have to improve reactivity and support decentralised organisation structures. To improve planning flexibility scheduling systems should interact better with process planning and make use of manufacturing alternatives. Finally, a continuous monitoring of workshop performance will be required as a first step towards a closed-loop workshop control.

Time and cost of manufacturing are significantly determined by the performance and degree collaboration of manufacturing planning, process planning and workshop scheduling. Today, process planning and workshop scheduling in industry are carried out as sequential, noncollaborative tasks. This has several disadvantages. The flexibility to react upon workshop disturbances is low. Sequential process planning and scheduling results in long through-put times. Generating process plans without considering actual workshop load and bottle-necks results in process plans which are not optimal for the situation in the workshop at the time of order execution. Moreover, the description of resources that are used by process planning and scheduling are often not consistent. The scheduler and shop floor control department can not call upon a process planner to assist whenever rescheduling also requires alternative manufacturing processes or routes to be searched for. This can be improved by making those activities partially concurrent or at least collaborative. The different information flows in conventional and concurrent process planning and scheduling are outlined in Figure 1. 

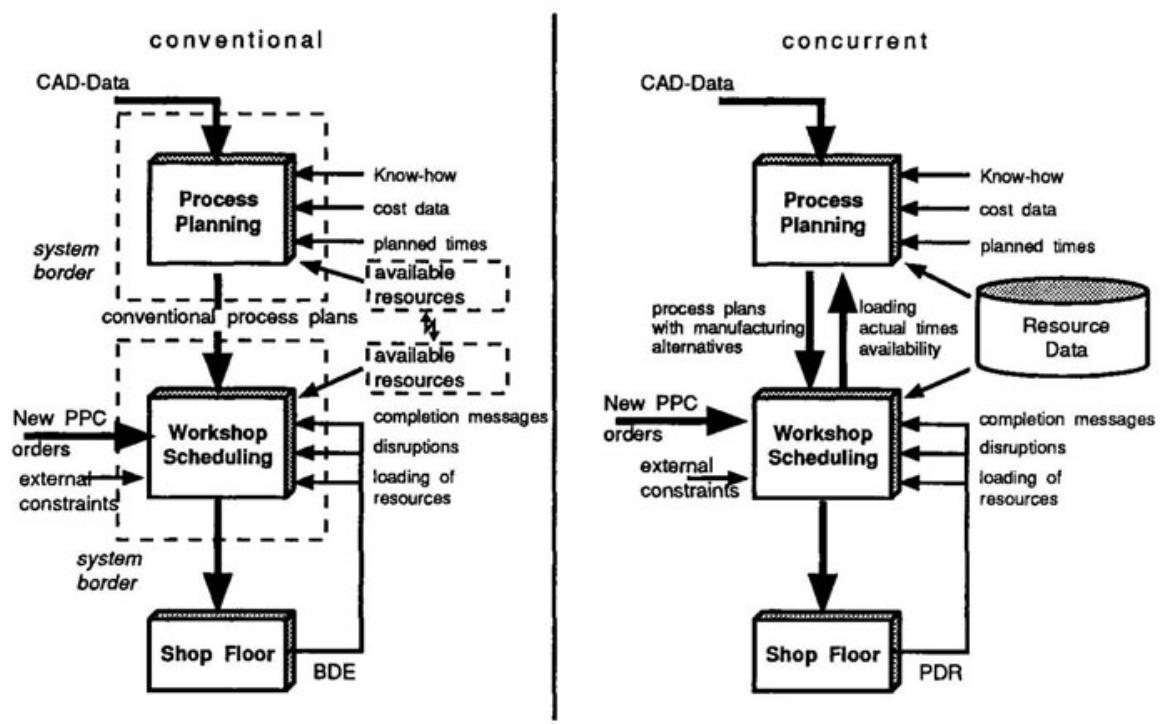

Figure 1 Flow of information between process planning and workshop scheduling

The integration or collaboration of scheduling and process planning has been the focus of extensive research efforts in recent years. A comprehensive overview of the world-wide efforts in this area is given by Elmaraghy (1993). An important research effort that has lead to a commercial system was performed in Twente (Houten 1991) where mainly the approach of dynamic, on-line process planning was pursued. In the COMPLAN project, only one order independent process plan is generated for each workpiece (Kreutzfeldt 1992). The flexibility for scheduling is improved by including manufacturing alternatives or possible changes in manufacturing sequence already in the process plan. The usually linear process plan sequence is enhanced to a net structure that contains the feasible operation steps. Several alternative routings or sequences of operations are combined in one Petri-net structure, so called nonlinear process plans (NLPPs). The most important feature of the COMPLAN process planning system is its ability to handle so called non-linear process plans. An example for such a netshaped process plan is given in Figure 2.

\subsection{Approach}

The target environment for the COMPLAN system is small batch production of complex products in a typical job shop environment. A certain complexity of the manufacturing process is required to be able to fully utilise the possible benefits of the system. Also, a certain level of computer support in design, order processing and workshop control should already exist in companies that aim at introducing the COMPLAN system. The following environment functions are required: 


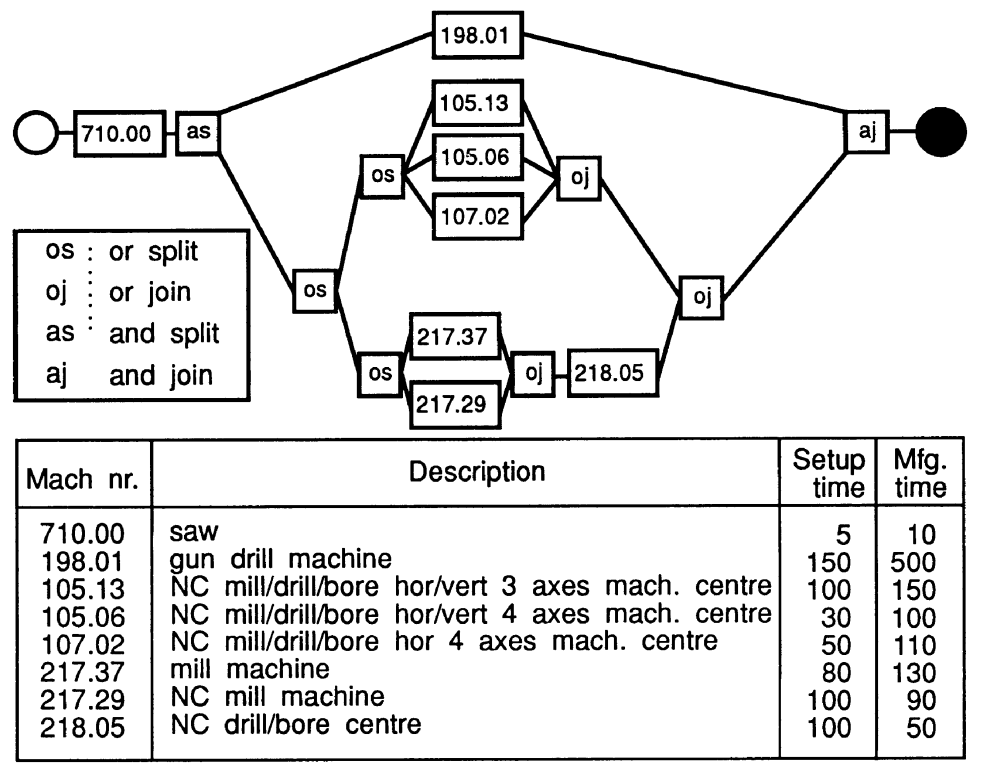

Figure 2 An example NLPP

A PPC system or a MRP system that performs the explosion of the bill of materials, performs material requirements planning, lot sizing and assigns due dates for the manufacturing or assembly of parts. This manufacturing order information is passed to the COMPLAN system. Manufacturing order information must comprise at least an identification for the workpiece to be produced, the quantity and the due date for the workpiece.

A design department that provides workpiece information for each workpiece. This information must comprise all information needed to create a process plan. On the one hand, an interface is foreseen towards wire-frame CAD systems, using a neutral programming interface.

The workshop must be able to provide production registration data. As a minimum, the workshop feedback data must contain the actual start- and finish dates of work orders.

The project is carried out under the framework and with funding from the ESPRIT program of the European Commission. The consortium consists of seven partners, where IPH acts as co-ordinating partner. AIS and CCI are commercial software developers. IPH is an independent non-profit research and consulting organisation in the area of computer applications in manufacturing that co-operates with industry and the University of Hannover. WTCM is the collective research centre of the metalworking industry in Belgium. KUL division PMA and IFW of the University of Hannover are well established university institutes in the area of production technology. LVD is a medium sized enterprise that develops and produces machine tools for sheet metal forming and cutting. LVD employs about 800 people.

This consortium structure ensures that innovative ideas are implemented in state-of-the-art software that considers requirements and constraints of typical industrial application. The 
system is currently implemented on different UNIX workstation platforms with OSF/Motif user interfaces. Developments are currently performed in parallel on DEC, HP, IBM and SUN workstations. The system is mainly developed in C++. LISP is used within the Petri Net Process Planning Module (PN PPM). It is implemented on an ORACLE database system.

\section{SYSTEM ARCHITECTURE}

The COMPLAN system provides the framework where all modules work together on the basis of a concurrent approach. The system supports a flexible link between the process planner and the workshop scheduler, resulting in a better overall performance in manufacturing. The whole system is based on an integrated relational manufacturing database. The modules of the COMPLAN system can be grouped in four main functional units as represented in Figure 3. The module acronyms use in the diagram are explained in the following.

\subsection{Process Planning (CPP)}

The Complan Process Planner has to perform two main functions. It has to handle the description of workpieces in features (Workpiece Delineator) as basis for automated process planning and it has to provide process planning functions for different situations and requirements.

These functions are controlled by so-called Editor Control Modules (ECMs). These modules feature graphical editors that allow to manually enter data about either the workpiece (WP ECM) or the non-linear process plan (NLPP ECM). The ECMs cover the entire workpiece spectrum and the complete set of process planning data of a company.

Additionally to the ECMs, the COMPLAN Process Planner is extended with a set of ,plugand-play" modules, allowing to obtain some of those data automatically or semi-automatically. These automatic modules are invoked and controlled from the editors.

The Workpiece Delineator (WPD) consists of a Workpiece Editor Control Module (WP ECM) allowing to manually enter workpiece data. To automatically obtain workpiece descriptions the WP ECM can call the CAD interface (CAD PPI) and the CODL file interface (CF PPI) modules that interpret CAD data.

Process planning functionality is provided by four modules: NLPP ECM, STS PPM, PN PPM, and AVOPLAN PPM. The NLPP Editor Control Module (NLPP ECM) offers the function to manually edit non-linear process plan data in a graphical editor. It also controls the State Space, the Petri Net and the AVOPLAN Process Planning Module. The STate Space Process Planning Module (STS PPM) and the Petri Net Process Planning Module (PN PPM) generate NLPPs automatically from feature based workpiece descriptions. The two modules apply different approaches to reach this goal. The AVOPLAN Process Planning Module (AVOPLAN PPM) generates process plans semi-automatically.

Getting the feature based workpiece description is the first step for automatic Process planning in COMPLAN. The Workpiece Editor Control Module (WP ECM) is able to edit manually a workpiece description and save it in the database. The WP ECM is also able to read the output of other Process Planning Interface (PPI) modules. 


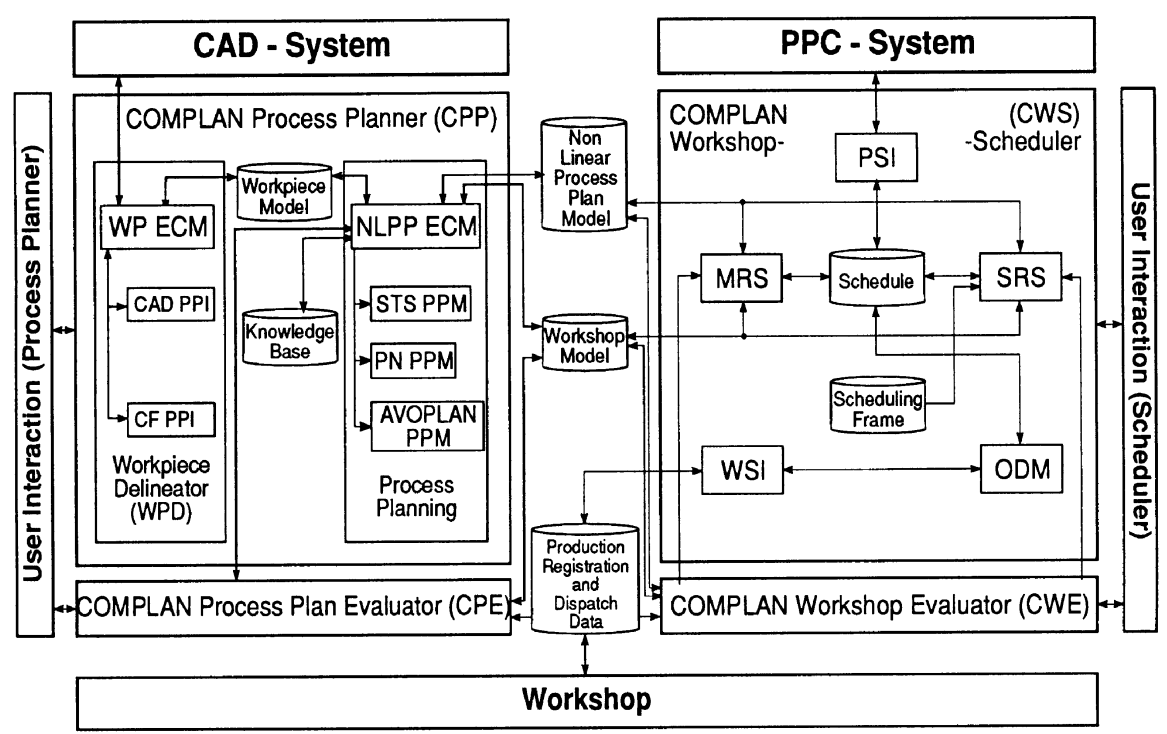

Figure 3 Architecture and Modules of the COMPLAN System

Using the CAD interface (CAD PPI), the feature-based description of a workpiece can be retrieved semi-automatically from 3-D wire frame CAD systems. This interface has been implemented for the Unigraphics system and for Applicon Bravo. For the ME 10 CAD system of LVD, a customised interface is being coded to overcome ME10 related problems. On the other hand, an interface is foreseen to feature-based CAD systems that write in standard file formats (so-called CODL-files).

Non-linear process plans (NLPPs) with manufacturing alternatives can be created or edited manually and be stored to the database with the graphical NLPP Editor Control Module.

Each of the automatic process planning modules can only be used for a limited spectrum of workpieces for which the feature catalogues and manufacturing constraints have previously been compiled. A workpiece testbed for different typical parts of LVD has been specified, and a feature catalogue has been compiled that specifies the process planning knowledge for each of the features used in those workpieces.

The PN PPM takes a feature based workpiece description as input and uses the concepts of Generic Petri nets, opportunistic process planning and constraint based search. The approach is described by Detand (1993) and Kruth et al. (1994). The current prototype supports all the steps of automatic process plan generation in a straightforward manner. The module is capable of generating fully automatically a NLPP for a simple LVD workpiece.

The State Space Process Planning Module uses a feature-based description of the workpiece that involves both geometrical and technological parameters. The current prototype supports an interactive generation of the operation steps of the NLPP. The STS PPM is linked to the ACIS modeller to allow geometric reasoning operations on the workpiece and features a graphical interface to display process planning information and a wire-frame model of the workpiece state. 
The AVOPLAN PPM is based on and covers the full functionality embedded in the existing commercial AVOPLAN system which is being re-engineered for the use in the COMPLAN environment. It provides powerful time- and cost calculation methods, and the generation of a complete process planning data using variant or similarity planning methods.

It is invoked from the NLPP ECM and supports variant planning for some LVD workpiece families, manual process planning of all those parts that cannot be handled by the automatic modules, and time and cost calculation for an NLPP generated by any module

Other modules supporting process planning could be added to enhance the system with even more automated or user specific functions at a later time.

All communication between CPP modules is based on the Complan Universal Process Planning Language (CUPPL) which describes a context free grammar for NLPP, workpieces, commands and constraints. The Complan Communication Server (CCS) makes it possible to synchronise different processes on multiple CPUs. The link from Process Planning to Scheduling is achieved by the information in the common database where all modules access the same central NLPP tables.

\subsection{Workshop Scheduling}

Based on the manufacturing orders coming from an external PPC or MRP system the COMPLAN Workshop Scheduler (CWS) creates a schedule that is dispatched to the workshop. The CWS applies a two-level hierarchical approach to scheduling with two levels of scheduling detail and scheduling horizon. It is distinguished between workcenter and workstation level, where workcenter basically comprises a group of workstations with very similar machining capabilities. The architecture of the COMPLAN Workshop Scheduler is shown in Figure 4.

The Production Planning and Control System Interface (PSI) provides a connection to the required upstream PPC or MRP system. It receives data from the external system or reads it from external files and transfers the relevant information to the COMPLAN data base.

Scheduling is then performed by two modules. The Medium Range Scheduling module (MRS) has a wider time horizon for scheduling and operates on an aggregated level of detail. In the Short Range Scheduling module (SRS), individual orders will be allocated to specific workstations. The time horizon of scheduling is limited and the actual state of the workshop is taken into account for the compilation of the schedule.

In hierarchical scheduling several modules, mainly the MRS, SRS and ODM, work on the same data objects (work orders). Therefore, interaction between these modules has to be organised. Each module needs information on which scheduling actions have already been performed, which actions need to be done and what the actual state of an order in the shop is. This is necessary to avoid that orders are rescheduled when they are already dispatched to the shop floor where order preparation might already been started.

The MRS has a wider time horizon for scheduling and operates on an aggregated level of detail. On this aggregated level of workcenters, the system performs capacity planning and follows a load-oriented strategy for load levelling and order release. The applied algorithm follows the method of Load-Oriented Order Release that was developed by the Institute for Production Systems (IFA) at the University of Hannover (Wiendahl 1994). It has been enhanced to consider and investigate NLPP information for manufacturing alternatives at bottleneck resources. One of the objectives of Complan is the early recognition of possible capacity bottlenecks. 
The output of the MRS are scheduled work orders with due dates and workcenters assigned to every operation in the process plan for the manufacturing of a workpiece. This Medium Range Schedule is the basis on which Short Range Scheduling is performed. Required input for Medium Range Scheduling are the manufacturing orders released by the external PPC or MRP system.

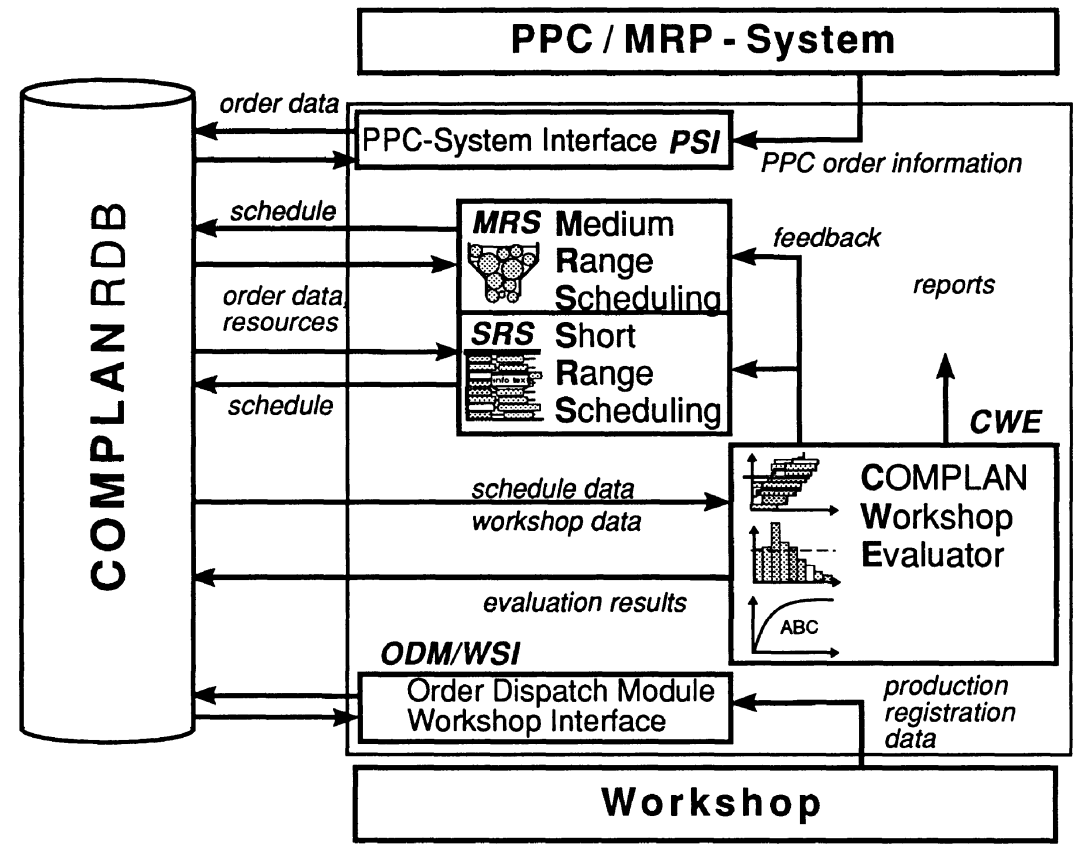

Figure 4 Architecture of the COMPLAN Workshop Scheduler

The current system mainly relies on user interaction for the selection of alternative routings and shall later be extended by automated strategies for selection of alternatives. The objective is to provide the SRS that operates with a shorter scheduling horizon with a rough scheduling basis to support and so to enable and improve more detailed scheduling decisions.

On the level of detailed scheduling (SRS), individual orders will be allocated to specific workstations. The SRS obtains the MR-schedule together with production registration data and returns the more detailed SR-schedule to the common data store. The returned SRschedule updates the load profile that is used as a planning basis in the next MR scheduling run.

To automatically generate schedules, an automated planning modules uses simulation methods to execute Petri Nets. The Petri Nets are compiled from the non-linear process plan nets from all orders that have been marked for automated scheduling. The SRS also provides a Gantt-Chart as graphical user interface that will allow manual scheduling or changing of every order by user interaction. Additionally to commercially available detailed scheduling systems, 
the SRS will be able to access and display information on manufacturing alternatives. Schedules can be manipulated interactively on a graphical planning board.

The Order Dispatch Module (ODM) and the Workshop Interface (WSI) connect COMPLAN to the workshop. The ODM generates auxiliary orders. Both auxiliary orders and work orders are dispatched individually following specific workshop needs. The ODM works automatically and communicates via the database with other modules. The Workshop Interface (WSI) provides the required feedback information from the workshop to the Complan system. The WSI delivers scheduled orders to the workshop of LVD and records feedback data. In addition to the WSI a module simulating workshop feedback has been developed for testing and presentation purposes.

\subsection{Process Plan Evaluator (CPE)}

The evaluation of process plans (CPE) aims at the quality improvement of process planning by evaluating workshop performance data. The technical reliability of process planning data is improved by collecting and evaluating statistical data on actual process and setup times versus planned times. The quality of NLPPs with respect to logistical criteria or the actual situation in the shop is improved by forwarding production constraints such as e.g. unavailability or overload of certain resources to interactive and automatic process planning modules. Also a messaging system will be implemented where e.g. schedulers can attach notes to objects of a process plan that shall later be read and considered by the interactive process planners.

\subsection{Production Evaluator (CWE)}

As the demands on PPC increase, the amount of data to be processed and interpreted has grown significantly. Smaller lot sizes and throughput times along with shorter planning cycles also contribute to this. PPC systems can only be significantly improved when employing closed-loop control strategies that use shop floor data as feedback information to derive meaningful reference values as planning parameters for PPC.

These deficiencies are mainly due to insufficient skill in processing, aggregating and interpreting shop floor data. Another reason is the frequently poor quality of feedback information with respect to correctness, completeness and actuality. Production managers are supplied with insufficient information to cope with the ever increasing, complex decision making in manufacturing management.

The COMPLAN Workshop Evaluator (CWE) is designed to perform evaluation of shop floor activities and provide the user with aggregated information, textual as well as graphical. The CWE mainly will be used interactively to support decision making by the scheduler. On demand the evaluation results are stored in the database for later use. Certain restricted functionality of the CWE is also available to other modules to provide a convenient access to relevant shop floor data. Since the user rarely wants to overview the whole workshop all evaluations and visualisations are based on a pre-selection of orders, resources and time horizons. apart from tables with a user dependent selection of characteristic values three types of diagrams are generated on demand.

Throughput diagrams will give detailed information about the progress of order flow (inand output), inventory, throughput time etc. and the related deviations of actual order data from the planned data respectively the scheduled times. 
Bar charts to generate e.g. load diagrams for displaying resource related information like the capacity profile versus the load profile or to display frequency distributions of e.g. order sizes.

General 2D diagrams for user definable axis are e.g. to generate the characteristic operation curve which displays output versus inventory levels.

The evaluation aggregates workshop performance data and provides graphical representations for a quick overview on the workshop situation. It makes it possible to evaluate the quality of a schedule and determine the workshop performance. Evaluation results support scheduling decisions, the choice of the appropriate scheduling strategy and provide feedback to the process planning department.

\section{INTEGRATION AT PILOT USER LVD}

The project aims at the development of an integrated software prototype, of which the main modules shall be operational and tested at the pilot end-user's site after 33 months of project runtime in May 1995. The COMPLAN software will be installed in the workshop for mechanical manufacturing of discrete parts. The LVD shop floor is a typical example of a job shop for small batch production (batches from 1 to 10). To execute the operations, LVD utilises mainly universal machining centres and general purpose tools. The production flow in the LVD job shop is rather arbitrary, and the bottle-neck resources are dynamically moving around. The NLPP concept is especially suited for this kind of production environments.

The generation of NLPPs (by the COMPLAN CAPP system) can be done automatically using the Petri Net or State Space Planning modules, semi-automatically using AVOPLAN variant and similarity planning, or manually in a graphical NLPP editor. The automated functions will only be worked out for a limited scope of workpieces. For hydraulic building blocks, that are an important part family at LVD, the knowledge base and manufacturing constraints have already been compiled and tested.

The modular structure of the COMPLAN system enables an integration of the system step by step in the existing data processing environment as well as a „sophisticated“ integration in future systems. In the start-up phase the first modules - mainly for the manual activities - will be installed and the COMPLAN data base will be set up. The scheduling modules (CWS) require the interfaces to PPC for the reception of manufacturing orders and to the data collection system for the feed back from the shop floor. Both interfaces can be realised by connection to the OnLine Process Control system (OLPC), which was developed by LVD for DNC functions and the collection of shop floor data.

\section{EFFECTS ON BUSINESSES}

Computer-aided process planning systems offer the last major and almost unused potential for a significant shortening of the throughput time from part design to part manufacturing. The growing demand for effective CAPP systems is caused by high effort in manufacturing planning required by complex $\mathrm{CNC}$-controlled manufacturing systems. The envisaged COMPLAN system would significantly reduce process planning time and effort. It will also provide additional flexibility for the workshop by effectively making manufacturing alternatives available. The project will provide the missing link between CAD and CAPP and will so 
contribute an important element towards a flexible and fully computer-aided process chain from design to NC-programming.

The current practice of rushed and improvised changes in the manufacturing routing endangers product quality and transparency of manufacturing costs. COMPLAN will support quality assurance efforts by providing process planning support to workshop operators. Together with an evaluation tool this will allow more rational decision making on the shop floor and will strengthen the competitiveness of small batch manufacturing of discrete part which is predominant in many SMEs.

\section{REFERENCES}

Detand, J. (1993) A Computer Aided Process Planning System Generating Non-Linear Process Plans, PhD Thesis, KU Leuven 1993.

Elmaraghy, Hoda A. et al. (1993) Evolution and Future Perspectives of CAPP. Annals of the CIRP Vol 42/2/1993.

Houten, F.J.A.M. van (1991) PART, a computer-aided process planning system. PhD Thesis, University of Twente, Enschede 1991

Kreutzfeldt, J. and Schmidt, B.(1992) Integrated Process Planning and Workshop Scheduling, Proceedings of the $8^{\text {th }}$ Int. IFIP WG5.3 Conference PROLAMAT 92, Tokio, June 23-26, 1992

Kruth, J.-P.; Detand, J.; Van Zeir, G.; Kempenaers, J; Pinte, J. (1994) Opportunistic Process Planning: A knowledge based technique for CAPP applications. $14^{\text {th }}$ ASME Int. Computers in Engineering (CIE94) Conf.; Minneapolis, September 11-14 1994

Lenderink, A. (1994) The integration of process and production planning in small batch manufacturing. PhD Thesis, University of Twente, Enschede 1994

Wiendahl, H.-P.(1994) Load Oriented Manufacturing Control, Springer, Berlin London 1994

\section{BIOGRAPHY}

Bernd C. Schmidt studied production technology at the University of Hannover, Germany and finished with a Master Degree (Dipl.-Ing.) in 1990. In 1987/1988 he spent a year as guest worker at the Automated Production Technology Division of the National Institute of Standards and Technology in Gaithersburg, Maryland, USA. In 1990 he joined the logistics department of the former CIM-Fabrik Hannover as assistant to Prof. Wiendahl. When CFH changed its name to $\boldsymbol{I P H}$-Institut für Integrierte Produktion Hannover in 1992 he became head of the logistics department, which today employs 8 research and project engineers. His main task is currently the co-ordination of the COMPLAN project. 